\title{
L'instauration de la pharmacovigilance dans un nouveau centre hospitalier universitaire au Maroc : comment et pourquoi ?
}

Y. Khabbal, ${ }^{1}$ L. Alami et C. Nejjari ${ }^{3}$

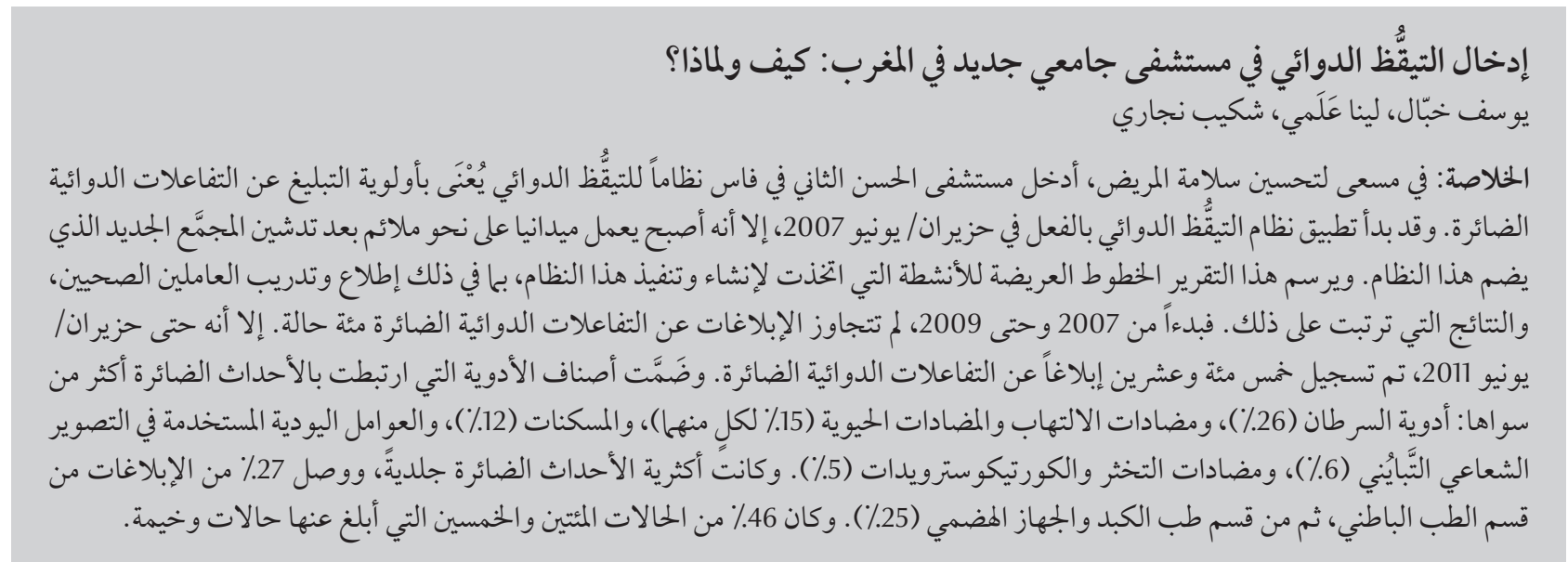

RÉSUMÉ Dans un souci d'améliorer la sécurité des patients, le CHU Hassan II de Fès a instauré un système de pharmacovigilance pour la notification des effets indésirables des médicaments en tant qu'objectif prioritaire. La mise en place de l'activité de pharmacovigilance a débuté effectivement en juin 2007 mais ce n'est qu'en 2009, après l'inauguration du nouveau complexe, que le système a connu l'essor qu'il mérite. Ce rapport présente les modalités de mise en place de ce système, et notamment la sensibilisation et la formation des professionnels de santé, ainsi que les résultats obtenus après son instauration. De 2007 à 2009, les notifications d'effets indésirables médicamenteux ne dépassaient pas une centaine de cas. Jusqu'à juin 2011 toutefois, 520 notifications spontanées d'effets indésirables ont été enregistrées. Les classes de médicaments les plus incriminées dans les effets indésirables sont les anticancéreux (26\%), les anti-inflammatoires et les antibiotiques (15\% chaque catégorie), les antalgiques (12\%), les produits de contraste iodé (6\%), les anticoagulants et les corticoïdes (5\%). Les principaux effets indésirables étaient dermatologiques et $27 \%$ des déclarations émanaient du service de médecine interne, suivi par celui d’hépato-gastro-entérologie (25\%). Sur 520 cas notifiés, 46 \% étaient des cas graves.

\section{Introduction of pharmacovigilance in a new university hospital in Morocco: how and why}

ABSTRACT In an effort to improve patient safety, Hassan II hospital in Fez introduced a pharmacovigilance system for notification of adverse drug reactions as a priority objective. The implementation of pharmacovigilance activities actually began in June 2007 but it was in 2009 after the inauguration of the new complex that the system became properly operational. This report outlines the activities carried out to develop and implement this system, including informing and training the health professionals, and the results obtained after its introduction. From 2007 to 2009 , fewer than 100 cases of adverse drug reactions were reported. Up to June 2011, however, 520 reports of adverse drug reactions were recorded. The classes of drugs most implicated in adverse events were: cancer drugs (26\%), antiinflammatory drugs and antibiotics (each 15\%), analgesics (12\%), iodinated contrast agents (6\%), and anticoagulants and corticosteroids (5\%). The main adverse events were dermatological and $27 \%$ of reports came from the internal medicine department, followed by hepatogastroenterology (25\%). Of the 520 cases reported, 46\% were severe.

'Service de Pharmacotoxicologie, Laboratoire central d'Analyses médicales ; ${ }^{2}$ Service de la Pharmacie centrale, CHU Hassan II Fès, Fès (Maroc) (Correspondance à adresserà Y. Khabbal : youssefkhabbal@gmail.com).

${ }^{3}$ Laboratoire d'Épidémiologie et de Santé Publique, Faculté de Médecine et de Pharmacie, Fès (Maroc).

Reçu : 26/12/11; accepté : 05/02/12 


\section{Introduction}

L'histoire de la pharmacovigilance internationale remonte à plus de trente ans, quand la Vingtième Assemblée de l'Organisation mondiale de la Santé adopta une résolution sur la création d'un système international de surveillance des effets indésirables des médicaments.

À ce jour, plus de 70 pays participent à ce programme. Le monde d'aujourd'hui est confronté à des défis bien différents de ceux rencontrés au moment de la création du programme. Les nouveaux développements exigent des réactions appropriées et soulèvent de nouvelles questions sur les modalités de surveillance des effets indésirables des médicaments [1].

À cet égard, et dans un souci d'améliorer la sécurité des patients, le Centre hospitalier universitaire (CHU) Hassan II de Fès a fait du développement del'activité de pharmacovigilance un de ses objectifs prioritaires.

Dans ce travail, nous présentons la stratégie que nous avons suivie et les résultats obtenus après la mise en place de notre système.

\section{Méthodes}

La création d'un système de pharmacovigilance efficace et futuriste nécessite une stratégie bien réfléchie et une mise en place solide [2].

L'activité de pharmacovigilance au CHU Hassan II de Fès a débuté effectivementenjuin2007.Ceprocessus de développement a été lancé dans les anciens locaux, ce qui a représenté une entrave à une approche plus engagée. Le principal objectif de cette étape, dont la chronologie est décrite ci-après, était de sensibiliser les professionnels de santé de notre établissement.

Mai 2007 : présentation et validation du projet. Nous avons présenté le projet de création d'un système local de pharmacovigilance avec une vision futuriste et en tenant compte des contraintes actuelles; ce projet a été validé parle comité des médicaments et des dispositifs médicaux du $\mathrm{CHU}$ Hassan II.

Juin 2007: sensibilisation des professionnels de santé. Au moment de sa création, des efforts importants ont été consacrés à la communication avec les professionnels de santé, surtout les médecins mais aussi les infirmiers et les étudiants en médecine, pour les sensibiliser à la notification spontanée; dans ce cadre, nous avons organisé une journée scientifique le 25 juin 2007. Cette journée a été animée par un médecin d'un Centre de Pharmacovigilance français et expert auprès de l'Agence française de sécurité sanitaire des produits de santé (AFSSAPS).

Juillet 2007 : réalisation d'une fiche de notification interne. Nous avons réalisé plusieurs propositions de fiche de déclaration dont le critère principal était que la notification soit pratique et réalisable en respectant les bonnes pratiques de pharmacovigilance [3]. Cette fiche a été validée par les responsables des services cliniques. Elle comporte essentiellement des données concernant les éléments suivants :

- le patient;

- l'effet indésirable;

- le(s) médicament(s) suspect(s);

- les autres médicaments associés (y compris ceux pris par automédication);

- les facteurs de risque ;

- le nom et l'adresse du notificateur.

Septembre 2007: désignation et formation des correspondants locaux de pharmacovigilance. Le principal obstacle que connaît chaque nouveau système en matière de déclaration est le remplissage de la fiche de notification, souvent perçue comme une contrainte administrative supplémentaire par les prescripteurs.

À cet égard, nous avons désigné et formé des correspondants dans chaque service clinique afin d'optimiser la notification avec plus de disponibilité et de proximité; ces interlocuteurs sont au nombre de 25 .

Janvier 2008: création de la Commission de pharmacovigilance et de pharmaco-épidémiologie de Fès (CPPEF). Cette commission sera chargée en particulier du bon fonctionnement du système de pharmacovigilance.

La CPPEF est formée de 11 membres représentant les services les plus concernés par la pharmacovigilance. Les services représentés sont les suivants: le département de l'épidémiologie, le service de gastro-entérologie, le service de médecine interne, le service de dermatologie, le service de pédiatrie, le service d'oncologie, le service d'endocrinologie, la division des affaires médicales et des soins infirmiers, le service de pharmacie centrale et le service de pharmacologie.

Mai 2008 : publication du premier bulletin d'information sur le médicament et la pharmacovigilance. Ce bulletin, sous forme d'un dépliant, est destiné principalement aux professionnels de santé de notre CHU. La majeure partie est consacrée aux actualités relatives au médicament et aux effets indésirables à l'échelle nationale et internationale.

Juin 2008: deuxième journée de la Pharmacovigilance. Cette deuxième journée scientifique avait été organisée avec la collaboration d'un centre de pharmacovigilance français et avec la participation du Centre antipoison et de pharmacovigilance du Maroc. Le thème principal de cette manifestation était l'iatrogénie médicamenteuse.

La mise en place de l'activité de pharmacovigilance au CHU Hassan II de Fès a débuté effectivement en juin 2007, mais c'est à partir de janvier 2009 
que le système a connu l'essor qu'il mérite après l'inauguration du nouveau complexe hospitalier par Sa Majesté le Roi Mohammed VI. Ce centre couvre les besoins médicaux d'une région très étendue de plus de quatre millions d'habitants (Régions Fès Boulemane, Meknès-Tafilalet et Taza-Al HoceimaTaounate) ; il a une capacité de 880 lits, répartis dans 42 services.

2009-2011 : durant cette période se sont déroulées les activités suivantes.

- 2009 - Premier séminaire-atelier pour la formation des médecins résidents : imputabilité médicamenteuse (méthode française) [4,5].

- 2 septembre 2010 - Journée de formation à l'hôpital Ibn Khatib, un hôpital régional qui ne dépend pas de notre CHU.

- 2010 - Deuxième séminaire-atelier pour la formation des médecins résidents : les toxidermies.

- 2010 - Troisième séminaire-atelier pour la formation des médecins résidents: les néphropathies médicamenteuses.

- 2011 - troisième journée de la Pharmacovigilance: pharmacovigilance de la reproduction.

\section{Résultats}

La consultation de notre base de données montre que depuis fin juin 2007 jusqu'à fin juin 2009, les notifications d'effets indésirables médicamenteux qui ont été enregistrées par notre unité ne dépassaient pas une centaine de cas. L'ensemble de ces déclarations émanait des praticiens de notre CHU. La méthode de notification pendant cette période était essentiellement spontanée et rarement active. En juin 2011, le nombre de déclarations est de 520 cas, alors que les notifications sont toutes spontanées ayant pour objectif plutôt une demande d'avis qu'une déclaration proprement dite.
Les fiches sont complètes, bien remplies et leur suivi est fait à $100 \%$.

Ces notifications concernaient 132 hommes (53\%). L'âge moyen des patients était de 40 ans (ET 22, extrêmes 1-80 ans).

Les classes de médicaments les plus incriminées dans les effets indésirables étaient les anticancéreux avec $26 \%$ des cas, suivis par les anti-inflammatoires non stéroïdiens (AINS) et les antibiotiques avec $15 \%$, les antalgiques $12 \%$, les produits de contraste iodé $6 \%$, les anticoagulants et les corticoïdes $5 \%$ (Figure 1).

Vingt-sept pour cent des déclarations émanaient du service de médecine interne ; le deuxième service était celui de l'hépato-gastro-entérologie avec $25 \%$ des déclarations. La répartition des cas selon les services déclarants est représentée dans la figure 2.

Un effet indésirable est qualifié de grave :

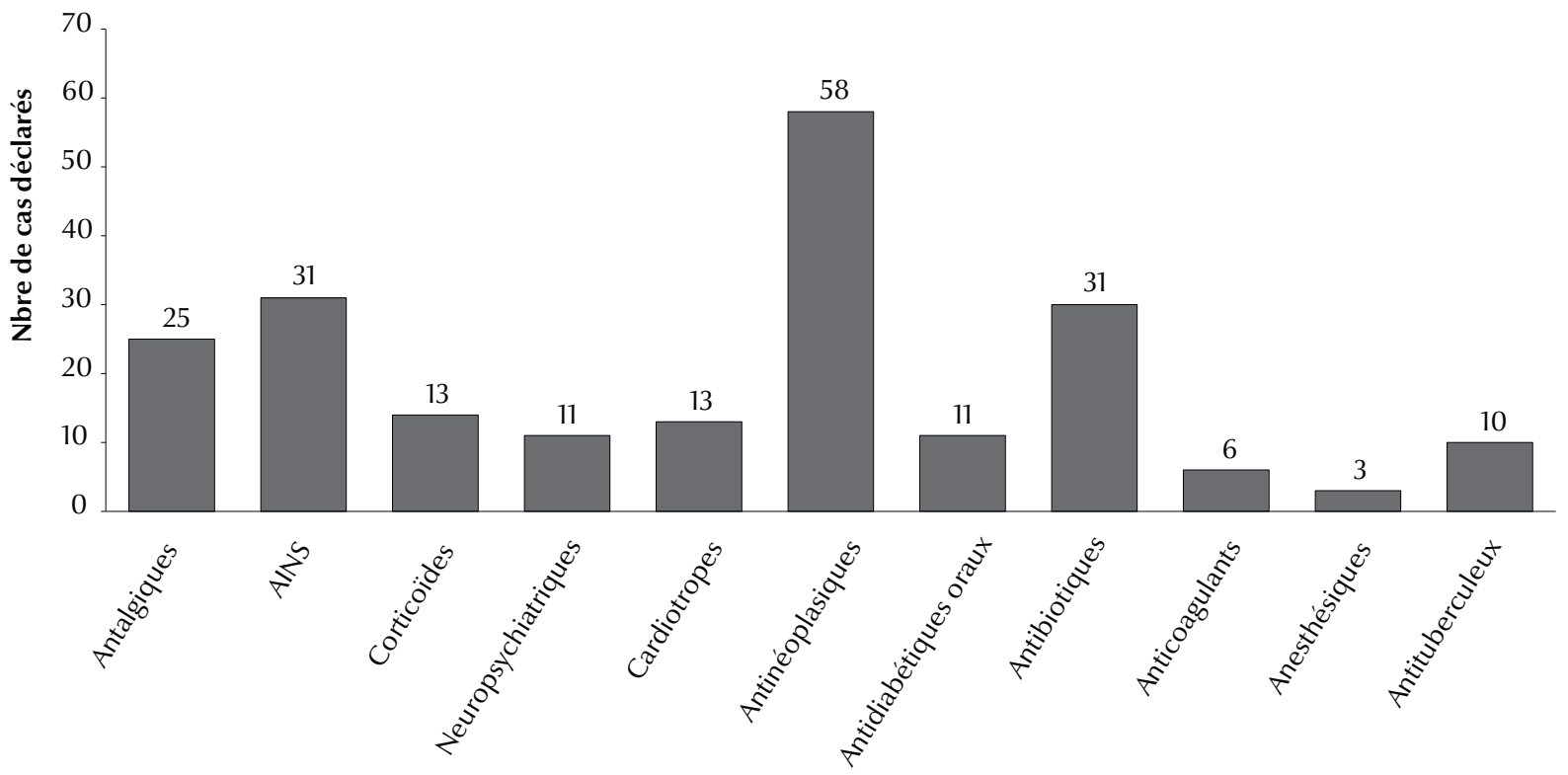

Familles thérapeutiques

Figure 1 Répartition selon les familles thérapeutiques. Note : les familles thérapeutiques ayant provoqué moins de trois effets ne sont pas représentées

AINS : anti-inflammatoires non stéroïdiens 


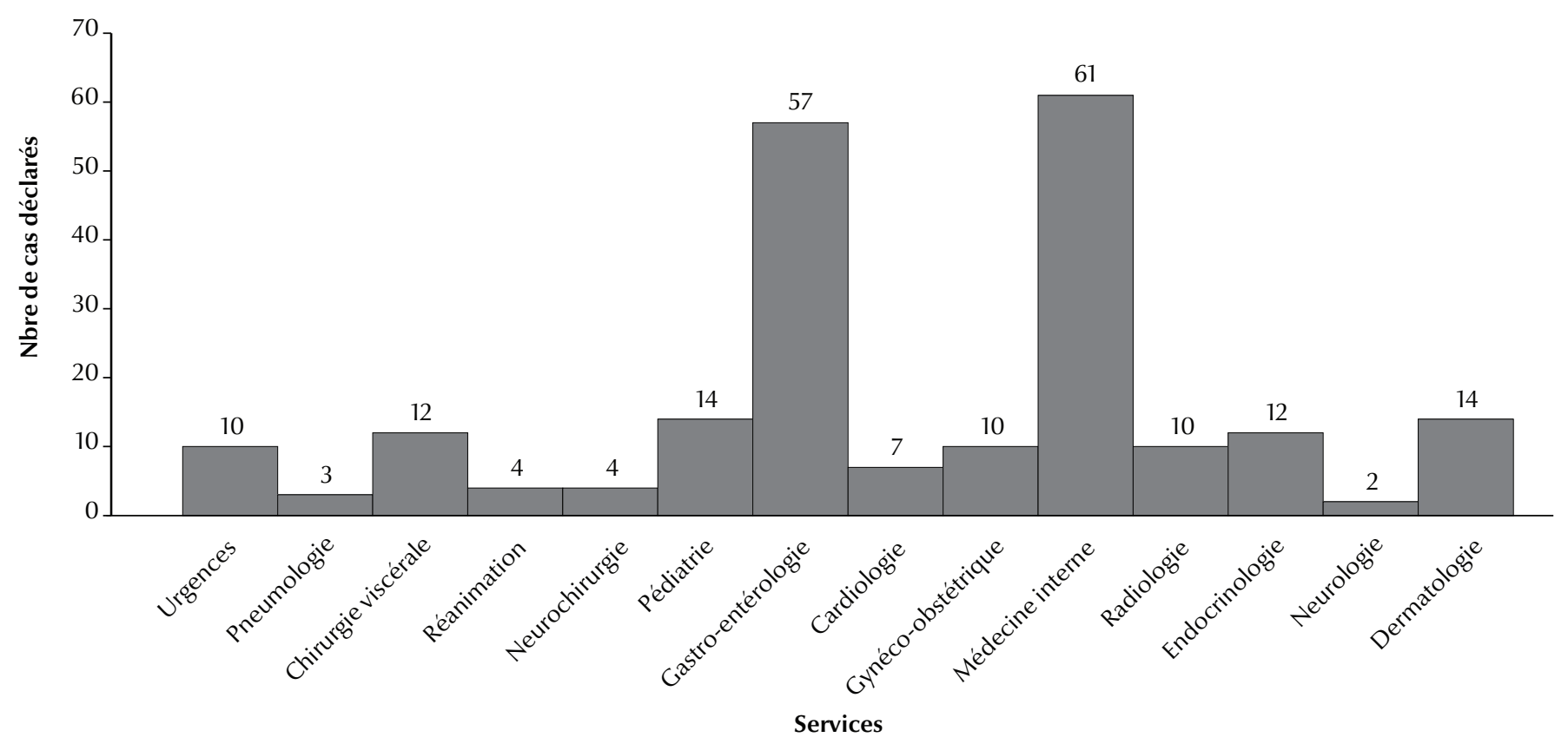

Figure 2 Répartition selon les services déclarants

- lorsqu'il entraîne un décès ou une invalidité ou une incapacité importante ou durable ;

- lorsqu'il provoque ou prolonge une hospitalisation ;

- lorsqu'il se manifeste par une anomalie ou une malformation congénitale $[6,7]$.

Sur 520 cas d'effets indésirables qui nous ont été notifiés, 46 \% étaient des cas graves.

\section{Discussion}

Lapharmacovigilance des médicaments mis sur le marché comporte un ensemble de techniques d'identification, d'évaluation et de prévention du risque d'effet indésirable des médicaments, que ce risque soit potentiel ou avéré.

La mise en place de plans de gestion des risques prolonge et élargit la démarche de pharmacovigilance pour certaines catégories de produits. Cette approche élargie de surveillance s'attache à identifier au maximum, avant la mise sur le marché, les enjeux et les méthodes de maîtrise des risques. De plus, elle intègre la notion d'évaluation constante du rapport bénéfice/risque dans les conditions réelles d'utilisation.

Les différents acteurs de l'évaluation du médicament vont donc: confirmer (ou infirmer), en situation réelle, l'efficacité du médicament; affiner et éventuellement étendre ou restreindre ses indications ( Vérification thérapeutique $\gg)$; recenser les effets indésirables et interactions médicamenteuses fâcheuses ; quantifier leur incidence et importance en pratique réelle établissant ainsi les critères d'innocuité («Pharmacovigilance»); et finalement étudier l'ensemble des conséquences médicoéconomiques imputables à l'usage du médicament («Pharmacoéconomie »).

- C'est à cet égard, et dans un souci d'amélioration de la sécurité des patients, que le CHU Hassan II de Fès a entamé une politique de gestion du risque. La pharmacovigilance représente donc une priorité de notre CHU. Dans notre établissement, et après plus de 4 ans d'activité, nous avons recueilli 520 cas d'effets indésirables. Ce résultat obtenu est déjà satisfaisant mais reste insuffisant vu le nombre annuel des hospitalisations et le nombre des médecins dans cet établissement. Selon une enquête française menée en 1997, $10 \%$ des patients hospitalisés en hôpital public, un jour donné, présentaient au moins un effet indésirable médicamenteux [8].

Le problème de la sous -notification est un phénomène commun à tous les pays ; le corriger est généralement difficile et sa portée peut être variable, voire méconnue. Même dans les centres bien établis, on estime que la notification des effets graves ne dépasse guère les $10 \%$. Par ailleurs, les premiers pays ayant participé au programme international de pharmacovigilance de l'OMS reçoivent en général 200 notifications par million d'habitants et par an, provenant de $10 \%$ des médecins. Dans beaucoup d'autres pays, cependant, les taux de notification sont bien inférieurs. 
La sous-notification peut retarder la détection de signal et amener à sousestimer l'ampleur d'un problème. Parmi les éléments qui peuvent participer à la sous-notification, on note :

- la crainte, pour certains professionnels de santé, de la remise en cause de leur compétence, voire la peur de répercussions médico-légales;

- la réticence à déclarer un cas d'effet indésirable quand ils ne sont pas certains du lien de causalité entre la manifestation de l'effet indésirable et le médicament (sachant qu'il est essentiel de déclarer toute réaction suspecte).

\section{Conclusion}

La mise en place d'un système de pharmacovigilance dans un nouveau centre hospitalier au Maroc témoigne de la volonté des praticiens à surveiller, évaluer et prévenir les risques médicamenteux potentiels ou avérés. C'est un moyen essentiel pour promouvoir le bon usage du médicament.

La régionalisation de la pharmacovigilance et la proximité des pharmacologues et/ou pharmaco-vigiles des praticiens hospitaliers facilitent la déclaration d'effets indésirables, et permettent d'apporter une aide et une information aussi efficaces que possible au prescripteur.

\section{Remerciements}

La réalisation de notre projet n'aurait pas été possible sans le travail professionnel des membres de la commission de la pharmacovigilance et leur implication également en tant que partenaires et collaborateurs. Cette commission se compose de personnes dynamiques visant l'excellence. Ces personnes à qui nous tenons à exprimer nos sincères remerciements sont les suivantes : Farida Ajdi, Wafaa Bono, Adil Ibrahimi, Ouadie Kendoussi, Mernissi Fatima Zahra, Omar El Mesbahi et Ouafae Mikou.

\section{Références}

1. Safety monitoring of medicinal products. Guidelines for setting up and running a pharmacovigilance centre. Uppsala, Uppsala Monitoring Centre, 2000.

2. Lebrun-Vignes B. La pharmacovigilance en 2004 : pour qui, pourquoi, comment? [Pharmacovigilance in 2004: why and how]? La revue de médecine interne, 2004, 25(7):487-489.

3. Bonnes pratiques de pharmacovigilance, édition décembre 1994 [Good practices in pharmacovigilance, December edition 1994]. Saint-Denis, Agence du Médicament, 1994.

4. Bégaud B et al. Imputabilité des effets inattendus ou toxiques des médicaments : actualisation de la méthode utilisée en France [Unexpected or toxic drug reaction assessment (imputation). Actualization of the method used in France ]. Thérapie, 1985, 40(2):111-118.

5. Montastruc JL et al. La pharmacovigilance et l'évaluation du risque médicamenteux : intérêt, fonctionnement et méthodes
[Pharmacovigilance for evaluating adverse drug reactions: value, organization, and methods]. Revue du rhumatisme, 2006, 73(10-11):1021-1024.

6. Décret $n^{\circ}$ 95-278 du 13 mars 1995 relatif à la pharmacovigilance et modifiant le code de la santé publique (deuxième partie : Décrets en Conseil d'État). Journal officiel de la République française $\mathrm{n}^{\circ} 62$ du 14 mars 1995, p. 3935.

7. Décret n 95-566 du 6 mai 1995 relatif à la pharmacovigilance exercée sur les médicaments dérivés du sang humain et modifiant le code de la santé publique (deuxième partie : Décrets en Conseil d'État). Journal officiel de la République française, 1995, $108: 7373$.

8. Imbs JL et al. latrogénie médicamenteuse : estimation de sa prévalence dans les hôpitaux publics français [latrogenic medication: estimation of its prevalence in French public hospitals]. Thérapie, 1999, 54(1):21-27. 